\title{
The Smell and Taste Corner: The Human Chemical Senses Are Useless, Are They Not?
}

\author{
Basile N. Landis ${ }^{a}$ Thomas Hummel ${ }^{b}$

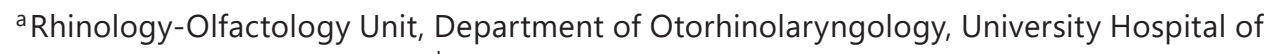

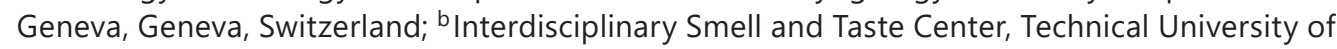 \\ Dresden, Dresden, Germany
}

Questioned about human senses, most people would spontaneously name vision, hearing, touch, and maybe smell. In contrast, taste is often forgotten, let alone the trigeminal sensitivity, and only few people are aware of the vestibular senses we have. So, in a way, people indicate that the chemical senses are useless. We would like to maintain that they are not loss of olfactory function is associated with loss of flavor, social competence, and depression [1].

With the exception of sight, all these senses are, not exclusively, but widely located in the area of otolaryngologists. During the specialty training, we spend huge efforts on acquiring surgical skills and management of diseases but often forget that we do work on sensory organs, and too little attention is paid to the physiologic comprehension of these systems. With this upcoming series of articles labeled as "Smell and Taste Corner," we would like to focus on the human chemical senses.

Humans have at least three systems that enable them to perceive aqueous or volatile molecules to be sensed. Olfaction, located in the nose, and taste in the oropharyngeal cavity, are probably familiar to most specialists. However, the third chemical sense, the trigeminal system, represents the touch/tactile and chemo-somatosensory system of the oral and nasal mucosa and is less known. Clinically underexplored, it is probably a major player in maintaining normal nasal and oral perception, with dysfunctions being very disabling, probably involved in empty nose [2] or burning mouth syndrome.

The field of chemical senses has also seen a tremendous evolution since it became clear that olfactory disorders precede many neurodegenerative disorders, opening a door to potential early, inexpensive, and easily accessible screening for such diseases [3]. Very recent contributions further suggest that olfactory function is highly trainable in health and disease [4], and that good olfactory function seems to be a reliable predictor for overall health and is inversely associated to mortality in the elderly population [5]. Consequently, smell and taste that are intimately related to otolaryngology because precise examination and testing has 
Landis and Hummel: The Smell and Taste Corner: The Human Chemical Senses Are Useless, Are They Not?

largely been developed in our field, have become more and more interesting to general medicine and neuroscience. The common ear-nose-throat (ENT) thinking that nothing can be done in smell and taste disorders and that only few ENT colleagues are active in the field needs to be readdressed. In the future, we the otolaryngologists will be more confronted with questions related to chemosensory disorders and we better be prepared. Despite the recent achievements in the field, many open questions regarding physiology, clinical impact, and treatment remain. As an example, distorted chemosensory perception still remains poorly understood and investigated [6].

The Journal for Otorhinolaryngology and Its Related Specialties initiates now a series called "Smell and Taste Corner," dedicated to contributions around the chemical senses to give more attention to and raise the awareness in this field of ENT that is still but wrongly neglected by most colleagues. By doing so, the Journal for Otorhinolaryngology and Its Related Specialties is, to the best of our knowledge, the first general ENT journal giving the chemical senses an own section. The very first contribution by Iannilli et al. [7] nicely provides new data regarding the central processing in patients with parosmia, a yet poorly understood condition.

\section{Disclosure Statement}

The authors have no conflicts of interest to disclose.

\section{References}

1 Hummel T, Whitcroft KL, Andrews P, Altundag A, Cinghi C, Costanzo RM, et al. Position paper on olfactory dysfunction. Rhinol Suppl. 2017 Mar;54 26:1-30.

2 Konstantinidis I, Tsakiropoulou E, Chatziavramidis A, Ikonomidis C, Markou K. Intranasal trigeminal function in patients with empty nose syndrome. Laryngoscope. 2017 Jun;127(6):1263-7.

3 Haehner A, Boesveldt S, Berendse HW, Mackay-Sim A, Fleischmann J, Silburn PA, et al. Prevalence of smell loss in Parkinson's disease-a multicenter study. Parkinsonism Relat Disord. 2009 Aug;15(7):490-4.

4 Sorokowska A, Drechsler E, Karwowski M, Hummel T. Effects of olfactory training: a meta-analysis. Rhinology. 2017 Mar;55(1):17-26.

5 Liu B, Luo Z, Pinto JM, Shiroma EJ, Tranah GJ, Wirdefeldt K, et al. Relationship Between Poor Olfaction and Mortality Among Community-Dwelling Older Adults: A Cohort Study. Ann Intern Med. 2019 Apr;170(10):673.

6 Landis BN, Reden J, Haehner A. Idiopathic phantosmia: outcome and clinical significance. ORL J Otorhinolaryngol Relat Spec. 2010;72(5):252-5.

7 Iannilli E, Leopold DA, Hornung DE, Hummel T. Advances in Understanding Parosmia: an fMRI Study. ORL J Otorhinolaryngol Relat Spec. DOI: 10.1159/000500558. 